\title{
Atualização \\ Insuficiência Cardíaca com Função Sistólica Preservada
}

\author{
Evandro Tinoco Mesquita, Jaderson Socrates, Salvador Rassi, Humberto Villacorta, Charles Mady \\ Rio de Janeiro, RJ
}

A insuficiência cardíaca é uma síndrome complexa, devida à incapacidade do coração de oferecer adequada oferta de oxigênio aos tecidos. A insuficiência cardíaca crônica usualmente manifesta-se por dispnéia, edema e fadiga.

Esta síndrome comumente evoca a imagem de um coração dilatado com reduzida função sistólica do ventrículo esquerdo. Porém, a insuficiência cardíaca não é uma entidade única e pode ocorrer em condições em que a função sistólica esteja deprimida (disfunção sistólica) ou com função sistólica preservada (disfunção diastólica) ${ }^{1}$.

A insuficiência cardíaca com função sistólica preservada não é um diagnóstico e sim uma síndrome clínica, onde está presente uma constelação de achados de insuficiência cardíaca associada a uma normal ou quase normal fração de ejeção do ventrículo esquerdo (> $50 \%$ ). Diversas etiologias estão envolvidas na insuficiência cardíaca com função sistólica preservada, sendo obrigatoriamente excluídas possíveis causas não cardíacas (doença pulmonar obstrutiva crônica, obesidade, anemia, hipotireoidismo, insuficiência renal, entre outras), que poderiam ocasionar os sintomas de dispnéia e ou intolerância ao esforç $0^{2}$. A insuficiência cardíaca com função sistólica preservada é freqüentemente associada à diminuição do relaxamento do ventrículo esquerdo e/ou à redução de sua complacência e, nesses casos, definida como insuficiência cardíaca diastólica. Os mecanismos patológicos envolvidos na disfunção diastólica podem atuar de maneira isolada ou em combinação e são, geralmente, exacerbados pela perda da contração atrial (fibrilação atrial).

A prevalência da insuficiência cardíaca com função sistólica preservada varia entre $30 \%$ a $50 \%$ nos estudos epidemiológicos que avaliam, na comunidade, pacientes com insuficiência cardía$\mathrm{ca}^{1,3-6}$. No perfil clínico da insuficiência cardíaca com função sistólica preservada observa-se uma maior frequêencia de mulheres, idosos e hipertensos e apresenta menor mortalidade que a insuficiência cardíaca com disfunção sistólica, porém, as taxas de hospitalização e re-hospitalização são semelhantes. A primeira descrição clínica da insuficiência cardíaca com função sistólica preservada

Universidade Federal Fluminense, Universidade Federal de Goias, Universidade Federal do Rio de Janeiro, Universidade de São Paulo e Hospital Pró-Cardíaco

Endereço para Correspondência: Dr. Evandro Tinoco Mesquita - Rua General Polidoro, 192 - Botafogo - 22280-000 - Rio de Janeiro - RJ E-mail: etmesquita@cardiol.br

Os autores declaram potencial conflito de interesse. Investigadores do estudo clínico I. Preserve (Sanofi-Synthelabo/Bristol Myers-Squibb)

Recebido para Publicação em 20/3/03 numa população de idosos foi feita por Topol e cols., em 1985. As evidências científicas sobre o impacto do tratamento medicamentoso da insuficiência cardíaca com função sistólica preservada sobre a qualidade de vida, alívio sintomático ou mortalidade são escassas e, no momento atual, seis ensaios clínicos multicêntricos estão em curso, avaliando os efeitos sobre a morbimortalidade, dos inibidores da ECA e ou ARA-ll nesta síndrome.

\section{Epidemiologia}

A insuficiência cardíaca é hoje uma condição pandêmica, e uma das prioridades entre as enfermidades crônicas da Organização Mundial de Saúde. Estima-se que quase dois milhões e meio de brasileiros, cinco milhões de americanos e 15 milhões em todo mundo sejam portadores de insuficiência cardíaca ${ }^{7}$. Apesar dos avanços na prevenção e tratamento das doenças cardíacas terem reduzido a mortalidade cardiovascular em muitos países ocidentais, a insuficiência cardíaca vem aumentando sua incidência e prevalência. A sobrevida após o diagnóstico permanece pequena, sendo de 1,7 a 3,2 anos para homens e mulheres, respectivamente. A mortalidade por insuficiência cardíaca nos Estados Unidos cresceu 127\% entre 1979 e 1997. 0 número de internações hospitalares aumentou de 377.000 casos, em 1979, para 957.000, em 19971.

A contribuição da insuficiência cardíaca com função sistólica preservada, na população de portadores de insuficiência cardíaca, tem sido avaliada melhor na última década. 0 estudo Rochester Epidemiology Project ${ }^{8}$ encontrou insuficiência cardíaca com função sistólica preservada, definida como fração de ejeção do ventrículo esquerdo $>50 \%$, em $43 \%$ dos casos incidentes de insuficiência cardíaca, no ano de 1991, na região de Olmsted, Minnesota. Outros dois estudos, o Framingham Heart Study e o Cardiovascular Health Study ${ }^{9}$ apresentaram uma prevalência de insuficiência cardíaca com função sistólica preservada (FEVE > 50\%) em 51\% e $52 \%$ dos casos de insuficiência cardíaca, respectivamente. No Cardiovascular Health Study ocorreram 597 novos casos, sendo que $60 \%$ apresentaram insuficiência cardíaca com função sistólica preservada no momento do diagnóstico. A prevalência da insuficiência cardíaca com função sistólica preservada aumenta significantemente com a idade ${ }^{10} \mathrm{e}$ seu prognóstico parece ser melhor que 0 da insuficiência cardíaca por disfunção sistólica. Pacientes com insuficiência cardíaca com função sistólica preservada apresentam elevada taxa de mortalidade quando comparados a indivíduos controles normais, para uma mesma faixa etária. A prevalência da insuficiência cardíaca com função sistólica preservada está relacionada à idade avançada e apresenta importante implicação 
epidemiológica, devido ao maior envelhecimento da população mundial, particularmente no Brasil, onde se estima que no ano de 2025 teremos a $6^{\text {a }}$ maior população de idosos do planeta.

\section{Fisiopatologia}

A insuficiência cardíaca é definida como incapacidade do coração de gerar um adequado débito cardíaco, para atender às necessidades metabólicas dos tecidos ou para alcançar esse objetivo, necessitar de elevadas pressões de enchimento do ventrículo esquerdo. Esta situação pode estar associada a disfunção sistólica ou a uma função sistólica preservada. A insuficiência cardíaca com função sistólica preservada é uma síndrome que, por definição, não é causada por uma anormalidade da contratilidade miocárdica (quadro I). Na ausência de causas, como valvopatias, arritmias, estados de alto débito cardíaco e disfunção isolada do ventrículo direito, a insuficiência cardíaca com função sistólica preservada é comumente resultado de disfunção diastólica e, nesses casos, referida como insuficiência cardíaca diastólica (quadro II). Normalmente, o ventrículo esquerdo apresenta uma fase de relaxamento ativo que se inicia um pouco antes do fechamento de valva aórtica e termina na fase do enchimento rápido. 0 relaxamento ativo consome ATP (aproximadamente $25 \%$ do $\mathrm{MVO}_{2}$ ), necessário para retirada ativa do cálcio do citoplasma para o interior do retículo sarcoplasmático. Portanto, o relaxamento é um processo ativo, dependente de energia, sendo precocemente alterado durante a isquemia miocárdica, conforme evidências em animais e humanos. 0 relaxamento miocárdico resulta em um importante declínio da pressão no ventrículo esquerdo, facilitando seu enchimento rápido, mesmo com pressão atrial normal ou baixa. Após a fase de enchimento rápido, segue-se o enchimento ventricular lento, dependente de propriedades passivas do miocárdio. A fase do enchimento lento é função da complacência da câmara ventricular. A redução da complacência do ventrículo esquerdo ocorre por vários mecanismos, incluindo a fibrose miocárdica, secundária ao envelhecimento cardíaco, isquemia, infarto, processos infiltrativos (amiloidose, sarcoidose e hemocromatose), podendo também ser devida à hipertrofia do ventrículo esquerdo secundária à hipertensão arterial sistêmica ou à estenose aórtica ${ }^{11,12}$. 0 relaxamento do ventrículo esquerdo anormal e/ou diminuição de sua complacência, promovem alteraç̃es na hemodinâmica cardíaca, de tal forma, que o débito cardíaco normal somente é obtido através do aumento das pressões de enchimento do ventrículo esquerdo. 0 coração com disfunção diastólica tem o seu débito sistólico mantido à custa do volume diastólico, de modo que uma redução pronunciada de pré-carga, pode ocasionar síndrome de baixo débito. 0 aumento da contribuição da contracão atrial para o enchimento ventricular é um importante mecanis-
Quadro I - Etiologias da insuficiência cardíaca com função sistólica preservada

Hipertrofia ventricular esquerda

Cardiopatia hipertensiva

Estenose aórtica

Cardiopatia isquêmica

Infarto agudo do miocárdico

Cardiopatia isquêmica crônica

Arritmias

Fibrilação atrial

Cardiomiopatias

Cardiomiopatia hipertrófica

Cardiomiopatias infiltrativas

Amiloidose

Sarcoidose

Hemocromatose

Doenças de armazenamento

Endomiocardiofibrose

Pós-radioterapia

Idiopática

Diabetes Mellitus

Obesidade mórbida

Valvopatias

Estenose aórtica

Insuficiência mitral

Estenose mitral

Pericardiopatias

Pericardite constrictiva

Tamponamento pericárdico

Síndrome de Alto Débito

Anemia

Beri-beri

Tireoitoxicose

Fístula arteriovenosa

mo de adaptação, reduzindo a pressão no interior do átrio esquerdo e no território venocapilar pulmonar. A perda da contração atrial, durante episódio de fibrilação atrial, pode ocasionar importante piora sintomática (congestão pulmonar) e de baixo débito cardíaco, em portadores de insuficiência cardíaca diastólica.

\section{Etiopatogenia da insuficiência cardíaca com função sistólica preservada}

A insuficiência cardíaca com função sistólica preservada deve ser compreendida como uma síndrome complexa, com um componente periférico, a vasculatura arterial, e um componente central, o miocárdio (vaso-miócito-interstício), acometidos frente a alguns estímulos agressores, como por exemplo a hipertensão arterial ${ }^{12}$. As alteracões dos componentes periféricos e central se interrelacionam, perpetuando o círculo vicioso, importante para o desenvolvimento da insuficiência cardíaca (fig. 1). Eventos clínicos cardiovasculares se associam freqüentemente à insuficiência cardíaca com

\section{Quadro II- Marcadores clínicos e ecocardiográficos de insuficiência cardiaca diastólica}

- Marcada elevação da pressão sangüínea (pressão sistólica > 160mmHg ou pressão diastólica > $100 \mathrm{mmHg}$ ) durante o evento de insuficiência cardíaca

- Ecocardiografia com hipertrofia do ventrículo esquerdo concêntrica, sem anormalidades da contratilidade das paredes do ventrículo esquerdo

- Taquiarritmia com um período de enchimento diastólico encurtado

- Precipitação do evento por infusão de pequena quantidade de fluído intravenoso

- Melhora clínica em resposta ao tratamento direcionado à causa de disfunção diastólica (como diminuir pressão sangüínea, reduzir freqüência cardíaca ou restaurar o mecanismo de contração atrial) 


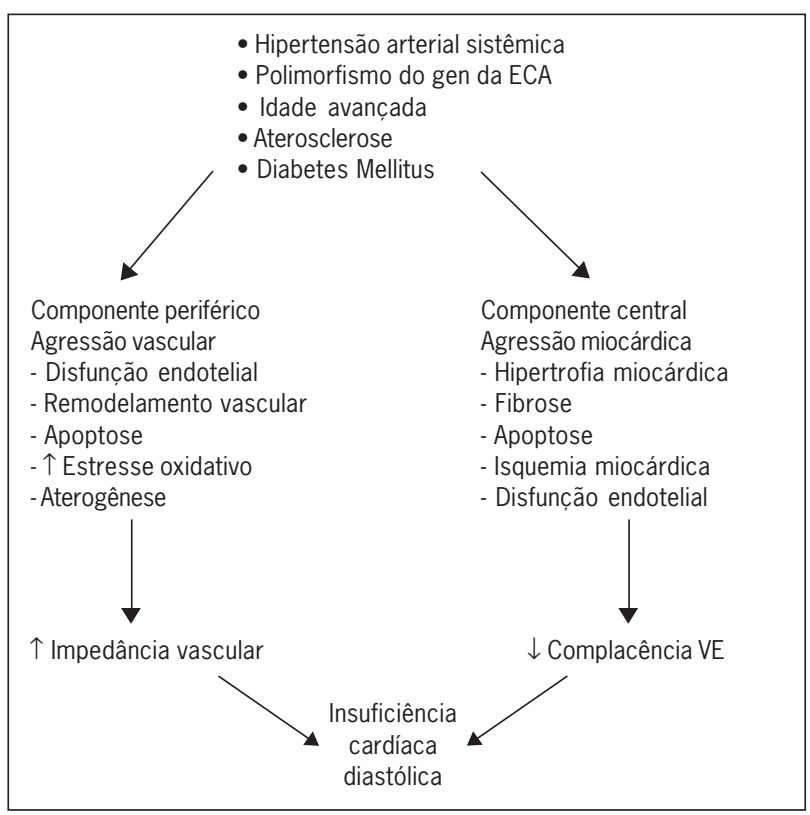

Fig. 1- Etiopatogenia da disfunção diastólica

função sistólica preservada, condições como infarto agudo do miocárdio, acidente vascular cerebral e fibrilação atrial, responsáveis pela sua elevada morbi-mortalidade. Várias evidências sugerem que 0 sistema renina-angiotensina-aldosterona possa ser um fator que contribua no desenvolvimento das anormalidades no componente periférico e central. Uma hiperatividade do sistema renina angiotensinaaldosterona contribui não somente para o desenvolvimento da hipertensão arterial, mas também para o relaxamento do miocárdio anormal e disfunção endotelial. Atualmente, acredita-se que a desmodulação e conseqüente hiperatividade do sistema renina angiotensina-aldosterona participem das complicações cardiovasculares (infarto agudo do miocárdio, acidente vascular cerebral, fibrilação atrial e morte súbita) presentes nos pacientes com a síndrome ${ }^{13-15}$.

\section{Relaxamento anormal}

Atualmente, novos conhecimentos moleculares vêm sendo incorporados à diastologia, particularmente envolvidos nas anormalidades do relaxamento miocárdico. A diminuição dos níveis ou da atividade da bomba cálcio ATP-ase do retículo sarcoplasmático (SERCA) pode ocasionar uma lenta remoção do cálcio citoplasmático do citosol. Níveis elevados ou aumento da atividade da fosfolamban, uma proteína natural inibidora da SERCA, pode também alentecer o relaxamento. 0 aumento dos niveis de AMPc, resultante da estimulação beta-1 adrenérgica ou da inibição da fosfodiesterase cardíaca, promove a fosforilação da fosfolamban e, desta forma, remove seu efeito inibitório sobre a SERCA, tornando o relaxamento mais rápido. A SERCA é uma enzima dependente de energia e a isquemia promove, desta forma, um alentecimento do relaxamento ativo. A hipertrofia patológica secundária à hipertensão arterial sistêmica ou à estenose aórtica resulta em redução da SERCA ou aumento do fosfolamban, contribuindo para o relaxamento anormal. A cardiomiopatia hipertrófica e o envelhecimento diminuem os níveis da SERCA. Estudos em animais demonstram que o captopril aumenta as ações da SERCA (ou diminui a ação

\section{Rigidez miocárdica}

0 ventrículo esquerdo tem uma complacência passiva e tornase mais rígido com o avanço da idade, o que parece ser devido, em parte, à fibrose difusa, conforme estudos patológicos e através da medida de marcadores seriais do metabolismo do colágeno. A rigidez passiva também está aumentada na presença de cicatriz focal ou de aneurisma após o infarto do miocárdio. Cardiomiopatias infiltratrativas, como a amiloidose, também aumentam a rigidez passiva $^{18}$.

\section{Fluxo microvascular}

Após a hipertrofia patológica ter ocorrido e a pressão diastólica do ventrículo esquerdo iniciar o seu aumento, a isquemia miocárdica pode ter um importante papel na piora da disfunção diastólica, mesmo sem estenose coronariana significativa. A compressão extravascular causada pela elevada pressão diastólica do VE que atua sobre capilares e pequenos vasos coronarianos de resistência, interfere com os mecanismos de regulação e a capacidade vasodilatadora. 0 turgor miocárdico resultante do ingurgitamento do sangue na microvasculatura, pode causar aumento da rigidez diastólica. Clinicamente isto ocorre em associação com a insuficiência cardíaca direita, quando as pressões elevadas do átrio direito levam a ingurgitamento venoso ${ }^{19,20}$.

\section{Avaliação diagnóstica da insuficiência cardíaca com função sistólica preservada}

Os pacientes com insuficiência cardíaca com função sistólica preservada podem apresentar-se de três formas: com síndrome de diminuição de tolerância aos esforços, como síndrome de retenção de líquidos ou apenas com achados de disfunção diastólica assintomática. É mais comum em mulheres e idosos e, em muitos casos, é difícil a caracterização dos sintomas como devida à insuficiência cardíaca, decorrente de comorbidades que podem ocasionar quadro clínico semelhante ${ }^{21,22}$. Os pacientes idosos e obesos, freqüentemente, apresentam doença pulmonar obstrutiva crônica, sedentarismo e varizes de membros inferiores, que podem simular sintomas observados na insuficiência cardíaca com função sistólica preservada. Na prática clínica, a primeira etapa é identificar se o sintoma do paciente é decorrente ou não da insuficiência cardíaca ${ }^{23}$. 0 eletrocardiograma pode apresentar sinais de hipertrofia ventricular esquerda e/ou sinais de áreas com inatividade elétrica. 0 achado de um eletrocardiograma completamente normal sugere a possibilidade de uma outra condição não cardíaca, para explicar a origem dos sintomas. A radiografia de tórax pode demonstrar sinais de hipertensão venocapilar e cardiomegalia. A dosagem do peptídeo natriurético do tipo $B$ vem sendo progressivamente incorporada à investigação de pacientes com suspeita de insuficiência cardíaca ${ }^{24}$. 0 nível de peptídeo natriurético do tipo B está elevado em pacientes com insuficiência cardíaca e, recentemente, também observado nos pacientes com insuficiência cardíaca com função sistólica preservada. Níveis superiores a $62 \mathrm{pg} / \mathrm{dl}$ traduzem especificidade e sensibilidade de cerca de 85\% para o diagnóstico de insuficiência cardíaca diastólica ${ }^{25}$. Portanto, 
o peptídeo natriurético do tipo B é útil para diferenciar a insuficiência cardíaca de outras condições que podem mimetizar seus sinais e sintomas (doença pulmonar obstrutiva crônica, obesidade e doença venosa crônica), particularmente no grupo de insuficiência cardíaca com função sistólica preservada, já que apresentam ecocardiograma com FEVE normal ${ }^{26}$. A próxima etapa diagnóstica é a identificação da anormalidade estrutural responsável pelo quadro de insuficiência cardíaca. Embora a história e o exame físico possam fornecer importantes informações sobre a natureza da anormalidade cardíaca de base, a identificação da anormalidade estrutural responsável pela insuficiência cardíaca requer, obrigatoriamente, um método de imagem e o método mais utilizado na avaliação de pacientes é a ecodopplercardiografia, hoje, método considerado indispensável na avaliação de todo paciente com suspeita da síndrome, conforme recentes diretrizes de Sociedades Internacionais, como a Sociedade Européia de Cardiologia, American College of Cardiology e American Heart Association ${ }^{23,27} \mathrm{e}$ também da diretriz de insuficiência cardíaca da Sociedade Brasileira de Cardiologia28 (fig. 2). Na avaliação ecocardiográfica, a pre- sença de fração de ejeção acima de 50\%, a despeito de evidenciar ou não diminuição do relaxamento ao Doppler transmitral, é hoje um critério não invasivo aceito para estabelecer o diagnóstico de insuficiência cardíaca diastólica, na presença de sinais e sintomas de insuficiência cardíaca ${ }^{11}$. 0 ecocardiograma é útil também na pesquisa de doenças cardíacas causadoras dos sintomas da síndrome e que podem cursar com fração de ejeção normal, como valvopatias aórtica e mitral hemodinamicamente significativas, e pericardiopatias. 0 cálculo da FEVE, pelo método de Simpson, é informação indispensável na avaliação ecocardiográfica de pacientes com suspeita de insuficiência cardíaca com função sistólica preservada. É sempre importante procurar a etiologia, porque algumas causas podem ser reversíveis ou tratáveis. A identificação e controle da hipertensão arterial, hipertireoidismo, anemia, insuficiência mitral transitória, estenose aórtica significativa, pericardite constrictiva e principalmente da isquemia miocárdica, assim como, a exclusão de doença pulmonar obstrutiva crônica e de insuficiência renal é parte fundamental da avaliação diagnóstica dos pacientes com insuficiência cardíaca com função sistólica preservada.

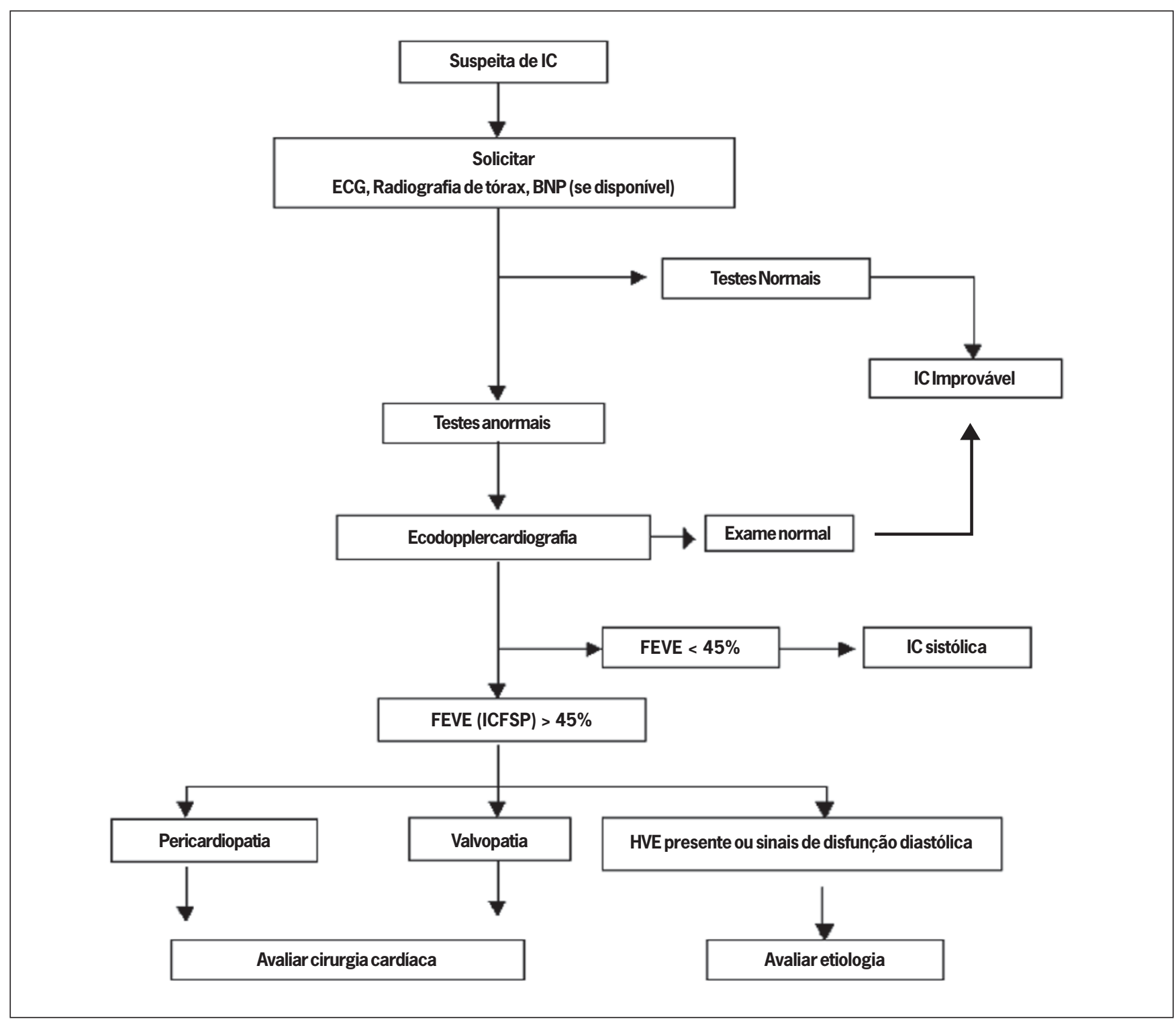

Fig. 2 - Algoritmo para avaliação diagnóstica da insuficiência cardíaca com função sistólica preservada. IC - insuficiência cardíaca; BNP - peptídeo natiurético B; FEVE - fração de ejeção do ventrículo esquerdo; ICFSP - insuficiência cardíaca com função sistólica preservada; HVE - hipertrofia ventricular esquerda. 
Vasan e Levy propuseram uma classificação de auxílio para sistematização do diagnóstico da insuficiência cardíaca diastólica, a ser aplicável a pacientes com insuficiência cardíaca, não relacionada a doença cardíaca valvular, cor pulmonale ou estado de sobrecarga de volume ${ }^{26}$, constando das seguintes fases: 1a) estabelecimento do diagnóstico, através de 1) sinais e sintomas compatíveis com a síndrome; 2) exames que confirmem o diagnóstico (ex.: radiografia de tórax); 3) resposta clínica típica à terapêutica com diuréticos; 2å) documentação objetiva da função sistólica do ventrículo esquerdo normal, em até $72 \mathrm{~h}$ do espisódio de insuficiência cardíaca, confirmada pela ecocardiografia ou ventriculografia radioisotópica a $F E V E \geq 50 \%$. E, finalmente, a busca da evidência objetiva de disfunção diastólica do ventrículo esquerdo, através do cateterismo cardíaco, observando índices anormais de relaxamento, enchimento e distensibilidade. A partir desses achados, os pacientes são classificados em: a) insuficiência cardíaca diastólica definitiva, b) insuficiência cardíaca diastólica provável e c) insuficiência cardíaca diastólica possível (tab. I).

0 diagnóstico de insuficiência cardíaca diastólica possível pode ser modificado para ICD provável, evoluindo desta forma para um nível de maior certeza, se a avaliação clínica for típica para a presença de disfunção diastólica de VE, demonstrado por um dos achados descritos no quadro II.

Esta classificação pode servir no futuro como uma ferramenta valiosa, que irá facilitar o desenho de ensaios clínicos apropriados, para testar a eficácia e especificidade de agentes terapêuticos no tratamento de insuficiência cardíaca diastólica. Critérios padronizados irão permitir a coleta de dados epidemiológicos para avaliar sua prevalência, história natural e prognóstico.

0 melhor método para definir a disfunção diastólica é o cateterismo esquerdo, através da avaliação das curvas de pressãovolume. Entretanto, esta metodologia é invasiva e não está disponível na maioria dos centros. Além disso, um recente estudo, utilizando dados invasivos e ecocardiográficos de disfunção diastólica, demonstrou que medidas objetivas da função diastólica podem ser dispensáveis para o diagnóstico de insuficiência cardíaca diastólica, na presença de sinais e sintomas de insuficiência cardíaca (avaliado no estudo pelos critérios de Framingham) e fração de ejeção acima de $50 \%{ }^{11}$. Assim, a simples medida da fração de ejeção pelo método de Simpson poderia substituir o dado invasivo do estudo hemodinâmico, para o diagnóstico de insuficiência cardíaca diastólica.

\section{Tratamento da insuficiência cardíaca com função sistólica preservada}

Embora existam evidências científicas sólidas, que muitos tratamentos possam melhorar a qualidade de vida e a sobrevida de pacientes com insuficiência cardíaca secundária à disfunção sistólica, há poucas evidências sobre o impacto sintomático ou prognóstico dos medicamentos na insuficiência cardíaca com função sistólica preservada. Diuréticos podem ser efetivos no controle de retenção hídrica. A angiotensina II tem efeitos deletérios sobre 0 relaxamento e o bloqueio do sistema renina-angiotensina melhora a distensibilidade diastólica do ventrículo esquerdo. Em pequeno estudo com verapamil, não ocorreu qualquer benefício na análise de subgrupo com esta síndrome. 0 tratamento com losartan promove melhora da função diastólica e da tolerância ao exercício, em hipertensos submetidos ao esforç ${ }^{29}$. 0 estudo LIFE demonstrou o benefício do losartan em comparação ao atenolol, em hipertensos portadores de hipertro-fia do ventrículo esquerdo, reduzindo a hipertrofia do ventrículo esquerdo, o aparecimento de acidente vascular cerebral e a mortalidade global. No estudo LIFE, porém, foram excluídos pacientes com insuficiência cardíaca e não se demonstrou redução do surgimento de novos casos em comparação com atenolo ${ }^{30}$. No estudo DIG, em um subestudo com 978 pacientes com FEVE $>45 \%$, ocorreu redução das taxas de hospitalização e dos sintomas ${ }^{31}$.

Atualmente, seis estudos multicêntricos, avaliando inibidores de enzina conversora, bloqueadores dos receptores de angiotensina II e betabloqueadores estão em andamento (tab. II). 0 estudo Preserve, o maior estudo nesta área, está sendo realizado com

\begin{tabular}{|lllll|}
\hline \multicolumn{4}{|c|}{ Tabela I-Classificação diagnóstica da insuficiência cardiaca diastólica (ICD) } \\
\hline & Evidência objetiva de IC & Evidência objetiva de função sistólica de VE normal & $\begin{array}{l}\text { Evidência objetiva de } \\
\text { disfunção diastólica do VE }\end{array}$ \\
\hline $\begin{array}{llll}\text { ICD definitiva } \\
\text { ICD provável }\end{array}$ & $\begin{array}{l}\text { Sim } \\
\text { ICD possível }\end{array}$ & Sim (dado obtido após 72h do evento clínico de IC) & $\begin{array}{l}\text { Sim (em proximidade ao evento de IC - em até 72h) } \\
\text { Sim (em até 72h do evento clínico informação de IC) }\end{array}$ & $\begin{array}{l}\text { Sim } \\
\text { inconclusiva } \\
\text { Informação inconclusiva }\end{array}$ \\
\hline
\end{tabular}

\begin{tabular}{|c|c|c|c|c|c|}
\hline Estudo & Comparada & Seguimento (n) & Critério diagnóstico & $\begin{array}{l}\text { Outros Importantes } \\
\text { Critérios Inclusão / Exclusão }\end{array}$ & Principais desfechos \\
\hline PEP-CHF & Placebo Perindopril & 1,00018 meses & $\begin{array}{l}\text { Critérios Clínicos e } \\
\text { ecocardiográficos }\end{array}$ & $\begin{array}{l}\text { Idade > } 70 \text { anos } \\
\text { diurético admissão }\end{array}$ & Morte e hospitalização por IC \\
\hline Charm-2 + & Placebo Candesartan & 2,50024 meses & $\mathrm{FE}>40 \%$ & Nenhum & Morte e hospitalização por IC \\
\hline I-Preserve & Placebo Irbersartan & $\begin{array}{l}3,600 \text { aprox. } \\
48 \text { meses }\end{array}$ & $\mathrm{FE}>45 \%$ & Diagnóstico clínico de IC & $\begin{array}{l}\text { Morte hospitalização por } \\
\text { doença cardiovascular }\end{array}$ \\
\hline Seniors & Placebo Nebivolol & $\begin{array}{l}2,000 \\
\text { (\% ICFS incerto) }\end{array}$ & $\begin{array}{l}\mathrm{FE}>35 \% \text { e anormalidade } \\
\text { cardíaca }\end{array}$ & $\begin{array}{l}\text { Idade }>70 \text { anos Hospital } \\
\text { admissão } 12 \text { meses }\end{array}$ & Morte e hospitalização \\
\hline Hong Kong & $\begin{array}{l}\text { Placebo Ramipril } \\
\text { Irbesartan }\end{array}$ & 45012 meses & Critério Doppler & Diuréticos & Morte ou hospitalização por IC \\
\hline Swedic & Placebo Carvedilol & 1409 meses & Critérios Doppler & Excluído FA & Regressão de disfunção diastólica \\
\hline
\end{tabular}


participação de 11 centros brasileiros e avaliará o impacto sobre a morbimortalidade do ibersartan em idosos com insuficiência cardíaca com função sistólica preservada nas classes III e IV (NYHA).

A abordagem terapêutica da insuficiência cardíaca com função sistólica preservada (quadro III) necessita de estudos clínicos randomizados, com casuística adequada, e, portanto, não temos disponíveis informações definitivas e embasadas em evidências. Desta forma, a conduta terapêutica está apoiada no racional fisiopatológico e na opinião de especialistas na área. Os quadros sintomáticos de congestão pulmonar, com ou sem sinais de congestão venosa sistêmica, podem ser tratados com suplementação de oxigênio, morfina, diurético parenteral e nitroglicerina endovenosa. 0 uso de diuréticos em altas doses pode resultar em grave hipotensão arterial, pois, com pequenas modificações do volume ventricular, podem ocorrer grandes modificações na relação pressãovolume diastólica do ventrículo esquerdo. Portanto, a terapia diurética deve ser iniciada em baixas doses. Caso o paciente esteja hipertenso, seus níveis tensionais devem ser normalizados. A isquemia miocárdica deve ser investigada e agressivamente tratada em todo paciente com insuficiência cardíaca com função sistólica preservada. Em pacientes com fibrilação atrial permanente, a restauração do ritmo sinusal e uma contração atrial organizada podem melhorar o enchimento diastólico e promover alívio sintomático.

No manuseio a longo prazo dos pacientes com insuficiência cardíaca com função sistólica preservada deve ser enfatizado: 1) tratar e prevenir o estado congestivo; 2) controle da hipertensão arterial; 3) avaliar e tratar a isquemia miocárdica; 4) manter o ritmo sinusal e prevenir taquicardia; 5) atuar bloqueando a ativação neuro-humoral e 6) melhorar a função diastólica.

Resumindo, a insuficiência cardíaca com função sistólica preservada não deve ser entendida como um diagnóstico ou uma síndrome, e sim como uma constelação de achados decorrente de diversas etiologias, onde são excluídas causas não cardíacas. Embora, critérios clínicos e ecocardiográficos ainda sejam imperfeitos, uma apresentação clínica de insuficiência cardíaca com função sistólica preservada comumente é indicativa de insuficiência cardíaca diastólica. A incorporação da dosagem do peptídeo natriurético tipo $B$ plasmático pode aumentar a acurácia diagnóstica. A disfunção diastólica é, portanto, uma doença vascular, com anormalidades miocárdicas, refletindo os efeitos mecânicos e tróficos, modulados pela hipertensão arterial crônica, reduzindo a complacência vascular. 0 tratamento da hipertensão arterial e
Quadro III - Abordagem terapêutica - insuficiencia cardíaca com função sistólica preservada

Reduzir os sintomas de congestão venosa / sistêmica

Restrição de sal e água

Diuréticos

Inibidor da enzima conversora e antagonistas do receptor de antiotensina II

Diálise

Controle da hipertensão arterial e promover a regressão da hipertrofia ventricular esquerda

Agentes anti-hipertensivos

Prevenir e tratar a isquemia miocárdica

Nitratos, beta-bloqueadores dos canais de cálcio, anti-trombóticos

Cirurgia de revascularização, angioplastia

Vastatinas

Prevenir taquicardia

Betabloqueadores, bloqueadores dos canais de cálcio

Ablação do nó atrioventricular e marcapasso

Manutenção da contração atrial (ritmo sinusal)

Agentes anti-arrítmicos

Marcapasso

Atenuar a ativação neuro-hormonal

Betabloqueadores, inibidor da enzina conversora

Prevenção de fibrose e promover a regressão da fibrose Inibidor da enzima conversora e antagonista do receptor de angiotensina II

Espironolactona

Agentes anti-isquêmicos

Melhora do relaxamento do ventrículo esquerdo

Betabloqueadores

Tratamento da isquemia miocárdica

Antagonistas do cálcio

da dislipidemia podem contribuir para prevenir a insuficiência cardíaca diastólica. Os medicamentos que modulam o sistema reninaangiotensina são particularmente atraentes, embasados nas anormalidades fisiopatológicos presentes nesta síndrome.

\section{Agradecimentos}

Este artigo é dedicado ao Prof. A. Carvalho Azevedo, membro fundador da SBC, recentemente eleito um dos 10 maiores cardiologistas brasileiros do século XX e um dos pioneiros, no nosso meio, na disseminação do conceito de insuficiência cardíaca diastólica.

\section{Referências}

1. Vasan RS, Larson MG, Benjamin EJ, Evans JC, Reiss C, Levy D. Congestive heart failure in subjects with normal versus reduced left ventricular ejection fraction: prevalence and mortality in a population-based cohort. J Am Coll Cardiol 1999; 33: 1948-55.

2. Caruana L, Petric MC, Davie AP, Mcmurray JJ. Do patients with suspected heart failure and preserved left ventricular systolic function suffer from diastolic heart failure or from misdiagnosis? Br Med J 2000; 321: 215-8.

3. Yip GWK, Ho PPY, Woo KS, Sanderson JE. Comparison of frequencies of left ventricular systolic and diastolic heart failure in chinese living in Hong Kong . Am J Cardiol 1999; 84: 563-7.

4. Dauterman KW, Massie BM, Georghiade M. Heart failure associated with preserved systolic function: a common and costly entity. Am Heart J 1998; 135 S: 310-S319.

5. Ceia F, Oliveira AG, et al-Epidemiologia da insuficiência cardíaca e aprendizagem. Rev Port Cardiol 1999; 18: 1151-5.

6. Mosterd $A$, Cost $B$ et al. The prognosis of heart failure in the general populationthe Rotterdam study. Eur J Heart Failure 2001; 22: 1318-27.

7. Senni M, Redfield MM. Heart failure with preserved systolic function - a different natural history? J Am Coll Cadiol 2001; 38: 1277-82.
8. Senni M, Tibouilloy CM, Rodeheffer RJ, et al. Congestive heart failure in the community: a study of all incident cases in Olmsted Country, Minnesota, in 1991. Circulation 1998; 98: 2282-9.

9. Gottdiener JS, Mc Clelland RL, Marshall R, et al. Outcome of congestive heart failure in elderly persons: influence of left ventricular systolic function. The Cardiovascular Health Study. Ann Intern Med 2001; 137: 631-9.

10. Maghert M, Persson H, Edner M, Kahan T. Epidemiology of heart failure in Sweden - a national survey. Eur heart J 2001; 3: 97-103.

11. Zile MR, Brutsaert DL. New concepts in diastolic dysfunction and diastolic heart failure: part l. Circulation 2002; 105: 1387-93.

12. Zile MR, Brutsaert DL. New concepts in diastolic heart failure: part II. Circulation 2002; 105: 1503-8.

13. Schunkert H, Jackson B, Tang SS, et al. Distribution and functional significance of cardiac angiotensin converting enzyme in hypertrophied rat hearts. Circulation 1993; 87: 1328-39.

14. Flesch M, Schiffer F, Zilk 0 , et al. Angiotensin receptor antagonism and angiotensin converting enzyme inibition improve diastolic dysfunction and $\mathrm{Ca}(2+)=$ 
ATPase expression in the sarcoplasmic reticulum in hypertensive cardiomyopathy. JHypertens 1997; 15: 1001-9

15. Yamamoto K, Masuyama T, Sakata Y, et al. Local neurohumoral regulation in the transition to isolated diastolic heart failure in hypertensive heart disease: absence of AT1 receptor down regulation and overdrive of the endothelin system. Cardiovasc Res 2000; 46: 421-32

16. Cain BS, Meldrum DR, Joo KS, et al. Human SERCA2a levels correlate inversely with age in senescent human myocardium. J Am Coll Cardiol 1998; 32: 458-67.

17. Del Monte F, Williams $E$, Lebeche $D$, et al. Improvement in survival and cardiac metabolism after gene transfer of sarcoplasmic reticulum $\mathrm{Ca}(2+)-$ ATPase in a rat model of heart failure. Circulation 2001; 104: 1424-9

18. Watanabe J, Levine MJ, Bellotto F, et al. Left ventricular diastolic chamber stiffness and intramyocardial coronary capacitance in isolated dog hearts. Circulation 1993; 88: 2929-40.

19. Stem S. Angina pectoris without chest pain: clinical implications of silent ischemia. Circulation 2002; 106: 1906-8.

20. Watanabe J, Levine MJ, Bellotto $F$ et al. Effects of coronary venous pressure on left ventricular diastolic distensibility. Circ Res 1990; 67: 923-32.

21. Banerjee P, Banerjee T, Khand A, et al. Diastolic heart failure: neglected or misdiagnosed? J Am Coll Cardiol 2002; 39: 138-41.

22. Grossman W. Defining diastolic dysfunction. Circulation 2000; 101: 2020-1.

23. European Study Group on Diastolic Heart Failure. How to diagnose diastolic heart failure. Eur Heart J 1998; 19: 990-1003.
24. Morroson LK, Harrison A, Drishnaswamy P, et al. Utility of a rapid B-natriuretic peptide assay in differentiating congestive heart failure from lung disease in patients presenting with dyspnea. J Am Coll Cardiol 2002; 39: 202-9.

25. Lubien E, De Maria A, Krishanaswamy P, et al. Utility of B-natriuretic peptide in detecting diastolic dysfunction: comparison with Doppler velocity recordings. Circulation 2002; 105: 595-601.

26. Vasan RS, Levy D. Defining diastolic heart failure: a call for standardized diagnostic criteria. Circulation. 2000; 101:2118-21.

27. Hunt AS, Baker DW, Chin MH, et al. ACC/AHA guidelines for the evaluation and management of chronic heart failure in the adult: executive summary. J Am Coll Cardiol 2001; 38: 2101-13

28. Revisão das II Diretrizes da Sociedade Brasileira de Cardiologia para o Diagnóstico e Tratamento da Insuficiência Cardíaca. Arq Bras Cardiol 2002; 79 (supl 3).

29. Warner JG Jr., Metzger DC, Kitzman DW, et al. Losartan improves exercise tolerance in patients with diastolic dysfunction and a hypertensive response to exercise. J Am Coll Cardiol 1999; 33: 1567-72.

30. Kjeldsen SE, Dahlof B, Devereux RB, et al. Effects of losartan on cardiovascular morbidity and mortality in patients with isolated systolic hypertension and left ventricular hypertrophy: a Losartan Intervention for Endpoint Reduction (LIFE) substudy. JAMA 2002; 288: 1491-8.

31. The Digitalis Investigation Group. The effect of digoxin on mortality and morbidity in patients with heart failure. N Engl J Med 1997; 336: 525-33. 